\title{
Unipotent group actions on del Pezzo cones
}

\author{
Takashi Kishimoto, Yuri Prokhorov and Mikhail Zaidenberg
}

\begin{abstract}
In [KPZ11b] we showed that for any del Pezzo surface $Y$ of degree $d \geqq 4$ and for any $r \geqq 1$, the affine cone $X=$ cone $_{r\left(-K_{Y}\right)}(Y)$ admits an effective $\mathbb{G}_{a}$-action. In particular, the group $\operatorname{Aut}(X)$ is infinite-dimensional. In this note we prove that for a del Pezzo surface $Y$ of degree $\leqslant 2$, the generalized cones $X$ as above do not admit any nontrivial action of a unipotent affine algebraic group.
\end{abstract}

\section{Introduction}

We are working over an algebraically closed field $\mathbb{k}$ of characteristic 0 . Let $Y$ be a smooth projective variety with a polarization $H$, where $H$ is an ample Cartier divisor. A generalized affine cone over $(Y, H)$ is the normal affine variety

$$
\operatorname{cone}_{H}(Y)=\operatorname{Spec} \bigoplus_{\nu \geqslant 0} H^{0}(Y, \nu H) .
$$

This variety cone $_{H}(Y)$ is the usual affine cone over $Y$ embedded in a projective space $\mathbb{P}^{n}$ by the linear system $|H|$ provided that $H$ is very ample and that the image of $Y$ in $\mathbb{P}^{n}$ is projectively normal.

In this paper we deal with a smooth del Pezzo surface $Y$ of degree $d$ and a pluri-anticanonical divisor $H=-r K_{Y}$ on $Y$, where $r \geqslant 1$; we then call cone $_{H}(Y)$ a del Pezzo cone. This is a usual cone if $r \geqslant 4-d$ (see, for example, [Dol12, Theorem 8.3.4]) and a generalized cone otherwise.

It is known [KPZ11b, 3.1.13] that for any smooth rational surface there is an ample polarization such that the associated affine cone admits an effective $\mathbb{G}_{a}$-action. Furthermore, for any del Pezzo surface of degree $\geqslant 4$ and for any $r \geqslant 1$, the corresponding del Pezzo cone cone ${ }_{-r K_{Y}}(Y)$ admits such an action (loc.cit), and the group generated by all these $\mathbb{G}_{a}$-actions is infinitely transitive off the vertex of the cone [Per11]. An effective $\mathbb{G}_{a}$-action exists also on affine cones over certain smooth rational Fano threefolds with Picard number 1 [KPZ11b, KPZ11a]. However, for del Pezzo surfaces of small degrees the consideration turns out to be more complicated. In this paper we investigate the cases $d=1$ and $d=2$. Our main result can be stated as follows.

Theorem 1.1. Let $Y$ be a del Pezzo surface of degree $d=K_{Y}^{2} \leqslant 2$. Then for any $r \geqslant 1$, there

Received 15 June 2013, accepted in final form 30 August 2013.

2010 Mathematics Subject Classification Primary 14R20, 14J45; Secondary 14J50, 14R05

Keywords: affine cone, del Pezzo surface, additive group, group action

This journal is (C) Foundation Compositio Mathematica 2014. This article is distributed with Open Access under the terms of the Creative Commons Attribution Non-Commercial License, which permits non-commercial reuse, distribution, and reproduction in any medium, provided that the original work is properly cited. For commercial re-use, please contact the Foundation Compositio Mathematica.

The first author was supported by a Grant-in-Aid for Scientific Research of JSPS No. 24740003. The second author was partially supported by RFBR grant No. 11-01-00336-a, the grant of Leading Scientific Schools No. 4713.2010.1, Simons-IUM fellowship, and AG Laboratory SU-HSE, RF government grant ag. 11.G34.31.0023. 


\section{Unipotent Group aCtions on DEL PeZzo CONES}

is no nontrivial action of a unipotent affine algebraic group on the del Pezzo cone

$$
X_{r}=\text { cone }_{-r K_{Y}}(Y)=\operatorname{Spec} A, \quad \text { where } \quad A=\bigoplus_{\nu \geqslant 0} H^{0}\left(Y,-\nu r K_{Y}\right) .
$$

As in [KPZ11a, KPZ11b], we use in the proof a geometric criterion for the existence of an effective $\mathbb{G}_{a}$-action on the affine cone $\operatorname{cone}_{H}(Y)$ (see [KPZ12] and Theorem 2.1 below). Recently, using this criterion, I. Cheltsov, J. Park and J. Won succeeded in proving [CPW13, Theorem 1.7] that the affine cone over a smooth cubic surface in $\mathbb{P}^{3}$ does not admit any effective $\mathbb{C}_{+}$-action. This answers a question of $\mathrm{H}$. Flenner and the third author [FZ03, Question 2.22] and confirms a conjecture that arises naturally from results of Section 4 in our previous paper [KPZ11b]. Summarizing, a del Pezzo cone of degree $d$ comports an effective $\mathbb{C}_{+}$-action if and only if $d \geqslant 4$.

From Theorem 1.1 and [CPW13, Theorem 1.7] we deduce the following corollary.

COROLlary 1.2. In the same notation as before, assume that $d \leqslant 3$ and $r \geqslant 4-d$, so that $X_{r}=$ cone $_{-r K_{Y}}(Y)$ is a usual del Pezzo cone. Then any algebraic subgroup $G \subset \operatorname{Aut}\left(X_{r}\right)$ is isomorphic to a subgroup of $\mathbb{G}_{m} \times \operatorname{Aut}(Y)$, where $\operatorname{Aut}(Y)$ is finite.

Proof. As follows from Theorem 1.1, $G$ is a reductive affine algebraic group (in fact, a finite extension of an algebraic torus). Now Lemma 2.3.1 and Proposition 2.2.6 in [KPZ11b] yield the relations

$$
G \hookrightarrow \operatorname{Lin}\left(X_{r}\right) \simeq \mathbb{G}_{m} \times \operatorname{Lin}(Y) \subset \mathbb{G}_{m} \times \operatorname{Aut}(Y),
$$

where the group $\operatorname{Aut}(Y)$ is finite, see [Dol12].

We suggest the following conjecture:

1.3. Conjecture. If $d \leqslant 3$, then for any $r \geqslant 4-d$, the full automorphism group Aut $\left(X_{r}\right)$ of the del Pezzo cone $X_{r}$ of degree $d$ is a finite extension of the multiplicative group $\mathbb{G}_{m}$.

Sections 2, 3, and 4 contain necessary preliminaries. Theorem 1.1 is proven in Section 5 . The proof proceeds as follows. Assuming to the contrary that there exists a nontrivial unipotent group action on $X_{r}=$ cone $_{\left(-r K_{Y}\right)}(Y)$, there also exists an effective $\mathbb{G}_{a}$-action on $X_{r}$. By Theorem 2.1 there is an effective $\mathbb{Q}$-divisor $D$ on $Y$ such that $D \sim_{\mathbb{Q}}-K_{Y}$ and $U=Y \backslash D \cong Z \times \mathbb{A}^{1}$, where $Z$ is a smooth rational affine curve. Such a principal open subset $U$ is called a $\left(-K_{Y}\right)$-polar cylinder in [KPZ11b]. One of the key points consists in an estimate for the singularities of the pair $(Y, D)$. More precisely, we consider the linear pencil $\mathscr{L}$ on $Y$ generated by the closures of the fibers of the projection $U \cong Z \times \mathbb{A}^{1} \rightarrow Z$. Letting $S$ be the last exceptional divisor appearing in the process of the minimal resolution of the base locus of $\mathscr{L}$, we compute the discrepancy $a(S ; D)$. Using this and some subtle geometric properties of the pair $(Y, D)$, we finally come to a contradiction.

\section{Criterion}

Let $Y$ be a projective variety and let $H$ be an ample Cartier divisor on $Y$. Recall [KPZ11b] that an $H$-polar cylinder in $Y$ is an open subset $U=Y \backslash \operatorname{supp}(D)$ isomorphic to $Z \times \mathbb{A}^{1}$ for some affine variety $Z$, where $D$ is an effective $\mathbb{Q}$-divisor on $Y$ such that $D \sim_{\mathbb{Q}} H$, that is, $q D$ and $q H$ are linearly equivalent integral divisors for some $q \in \mathbb{N}$. Corollary 3.2 in [KPZ12] provides the following useful criterion for the existence of an effective $\mathbb{G}_{a}$-action on the affine cone (cf. also [KPZ11b, 3.1.9]). 


\section{Kishimoto, Prokhorov and Zaidenberg}

Theorem 2.1. Let $Y$ be a normal projective algebraic variety with an ample polarization $H \in$ $\operatorname{Div}(Y)$, and let $X=\operatorname{cone}_{H}(Y)$ be the corresponding generalized affine cone. If $X$ is normal, then $X$ admits an effective $\mathbb{G}_{a}$-action if and only if $Y$ contains an $H$-polar cylinder.

We apply this criterion to a del Pezzo surface $Y$ of degree $d \leqslant 2$ and a generalized cone

$$
X_{r}=\operatorname{Spec} \bigoplus_{\nu \geqslant 0} H^{0}\left(Y,-\nu r K_{Y}\right)
$$

associated with $H=-r K_{Y}$, where $r \geqslant 1$. It follows, in particular, that if the cone $X_{r}$ admits an effective $\mathbb{G}_{a}$-action, then $Y$ contains a cylinder $Y \backslash \operatorname{supp} D$ with $D \sim_{\mathbb{Q}}-K_{Y}$. This assumption finally leads to a contradiction, which proves Theorem 1.1.

Remark 2.2. In [KPZ11a, KPZ11b, KPZ12] we used different notions of an $H$-polar cylinder. In fact, in our setting these definitions are equivalent.

Indeed, let $Y, H$ be as in Theorem 2.1, and let $U=Y \backslash \operatorname{supp} D_{i}$, where $D_{i}$ for $i=1,2,3$, are effective $\mathbb{Q}$-divisors on $Y$. Consider the following conditions:

(1) $D_{1} \in|d H|$ for some $d \in \mathbb{N}$;

(2) $\left[D_{2}\right] \in \mathbb{Q}_{+}[H]$ in $\operatorname{Pic}_{\mathbb{Q}}(Y)$;

(3) $D_{3} \sim_{\mathbb{Q}} H$.

Obviously, if for some $i \in\{1,2,3\}$, there exists a $D_{i}$ satisfying (i), then for the remaining $j \in\{1,2,3\}, j \neq i$, there also exist $D_{j}$ satisfying $(\mathrm{j})$.

\section{Preliminaries on weak del Pezzo surfaces}

A smooth projective surface $Y$ is called a del Pezzo surface if the anticanonical divisor $-K_{Y}$ is ample, and a weak del Pezzo surface if $-K_{Y}$ is big and nef. The degree of such a surface is $\operatorname{deg} Y=K_{Y}^{2} \in\{1, \ldots, 9\}$.

Lemma 3.1 (see, for example, [Dol12, Proposition 8.1.23]). Blowing up a point on a del Pezzo surface of degree $d \geqslant 2$ yields a weak del Pezzo surface of degree $d-1$.

Theorem 3.2 (see, for example, [Dol12, Thm. 8.3.2]). Let $Y$ be a del Pezzo surface of degree $d$. Then the following hold.

(i) If $d \geqslant 3$, then $\left|-K_{Y}\right|$ defines an embedding $Y \hookrightarrow \mathbb{P}^{d}$.

(ii) If $d=2$, then $\left|-K_{Y}\right|$ defines a double cover $\Phi: Y \rightarrow \mathbb{P}^{2}$ branched along a smooth curve $B \subset \mathbb{P}^{2}$ of degree 4 .

(iii) If $d=1$, then $\left|-K_{X}\right|$ is a pencil with a single base point, say $O$. The linear system $\left|-2 K_{Y}\right|$ defines a double cover $\Phi: Y \rightarrow Q^{\prime} \subset \mathbb{P}^{3}$, where $Q^{\prime}$ is a quadric cone with vertex at $\Phi(O)$. Furthermore, $\Phi$ is branched along a smooth curve $B \subset Q^{\prime}$ cut out on $Q^{\prime}$ by a cubic surface.

The Galois involution $\tau: Y \rightarrow Y$ associated with the double cover $\Phi$ is a regular morphism. It is called a Geiser involution in the case $d=2$ and a Bertini involution in the case $d=1$.

Remark 3.3. Recall the following facts (see, for example, [Dol12]). For an irreducible curve $C$ on $Y$ we have $C^{2} \geqslant-1$ if $Y$ is a del Pezzo surface and $C^{2} \geqslant-2$ if $Y$ is a weak del Pezzo surface. In both cases $C^{2}=-1$ if and only if $C$ is a $(-1)$-curve, that is, if and only if $-K_{Y} \cdot C=1$, and $C^{2}=-2$ if and only if $C$ is a $(-2)$-curve, that is, if and only if $-K_{Y} \cdot C=0$. A weak del Pezzo surface is del Pezzo if and only if it has no $(-2)$-curve. 


\section{Unipotent group aCtions on Del PeZzo CONES}

If $d \geqslant 2$, then any curve $C$ on $Y$ with $-K_{Y} \cdot C=1$ is an irreducible smooth rational curve by statements (i) and (ii). By the adjunction formula such a $C$ must be a $(-1)$-curve.

Lemma 3.4. Let $Y$ be a del Pezzo surface of degree $d \leqslant 2$. Then any member $R \in\left|-K_{Y}\right|$ is reduced and $p_{a}(R)=1$. Moreover, $R$ is irreducible except in the case where

$-d=2 ; R=R_{1}+R_{2} ; R_{i}^{2}=-1$ for $i=1,2 ; R_{1} \cdot R_{2}=2$; and $R_{2}=\tau\left(R_{1}\right)$.

Furthermore, $\operatorname{Sing}(R) \subset \Phi^{-1}(B)$ and for any $P \in \Phi^{-1}(B)$, there is a unique member $R \in\left|-K_{Y}\right|$ that is singular at $P$.

Proof. We have $p_{a}(R)=1$ by adjunction. Let $R_{1} \varsubsetneqq R$ be a reduced irreducible component. Then $\left(-K_{Y}\right) \cdot R_{1}<\left(-K_{Y}\right) \cdot R=d$ and so $d=2$ and $R_{1}$ is a $(-1)$-curve by Remark 3.3. Since $R^{2}=d=2$, we have $R \neq 2 R_{1}$. Therefore $R=R_{1}+R_{2}$, where the $R_{i}(i=1,2)$ are $(-1)$-curves and $R_{1} \cdot R_{2}=\frac{1}{2}\left(R^{2}-R_{1}^{2}-R_{2}^{2}\right)=2$. Finally, in both cases we have $R=\Phi^{-1}(L)$, where $L$ is a line in $\mathbb{P}^{2}$. Thus $R$ is singular at $P$ if and only if $\Phi(P) \in B$ and $L$ is tangent to $B$ at $\Phi(P)$.

Remark 3.5. Let $R_{1}$ and $R_{2}$ be $(-1)$-curves on a del Pezzo surface $Y$ of degree 2 such that $R_{1} \cdot R_{2} \geqslant 2$. Then $R_{2}=\tau\left(R_{1}\right), R_{1} \cdot R_{2}=2$, and $R_{1}+R_{2} \in\left|-K_{Y}\right|$. Indeed, $R_{1}+\tau\left(R_{1}\right) \sim-K_{Y}$. Hence $\tau\left(R_{1}\right) \cdot R_{2}=-1$ and so $\tau\left(R_{1}\right)=R_{2}$.

\section{4. $(-K)$-polar cylinders on del Pezzo surfaces}

Here we adjust some lemmas of [KPZ11b, $\S 4]$ to our setting.

Notation 4.1. Let $Y$ be a del Pezzo surface of degree $d$. Suppose that $Y$ admits a $\left(-K_{Y}\right)$-polar cylinder

$$
U=Y \backslash \operatorname{supp}(D) \cong Z \times \mathbb{A}^{1} \text { with } \quad D=\sum_{i=1}^{n} \delta_{i} \Delta_{i} \sim_{\mathbb{Q}}-K_{Y},
$$

where the $\Delta_{i}$ are prime divisors, the $\delta_{i}>0$ are rational numbers, and $Z$ is a smooth rational affine curve. We let $\mathscr{L}$ be the linear pencil on $Y$ defined by the rational map $\Psi: Y \rightarrow \mathbb{P}^{1}$ which extends the projection $\operatorname{pr}_{1}: U \cong Z \times \mathbb{A}^{1} \rightarrow Z$.

Resolving, if necessary, the base locus of the pencil $\mathscr{L}$, we obtain a diagram

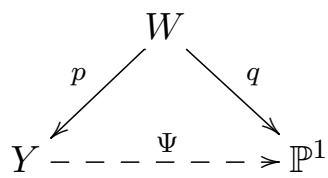

where we let $p: W \rightarrow Y$ be the shortest succession of blowups such that the proper transform $\mathscr{L}_{W}:=p_{*}^{-1} \mathscr{L}$ is base point free. Let $S$ be the last exceptional curve of the modification $p$ unless $p$ is the identity map, that is, $\operatorname{Bs} \mathscr{L}=\emptyset$. Notice that $S$ is a unique $(-1)$-curve in the exceptional locus $p^{-1}(P)$ and a section of $q$. The restriction $\left.\Phi_{\mathscr{L}_{W}}\right|_{U}$ is an $\mathbb{A}^{1}$-fibration and its fibers are reduced, irreducible affine curves with one place at infinity, situated on $S$.

LEMma 4.2. One of the following holds.

(i) Bs $\mathscr{L}$ consists of a single point, say $P$;

(ii) Bs $\mathscr{L}=\emptyset$ and $5 \leqslant d \leqslant 8$.

Proof. Since the general members of $\mathscr{L}$ are disjoint in $U$ and each one meets the cylinder $U$ along an $\mathbb{A}^{1}$-curve, Bs $\mathscr{L}$ consists of at most one point, which we denote by $P$. Suppose that 


\section{Kishimoto, Prokhorov and Zaidenberg}

Bs $\mathscr{L}=\emptyset$. Then the pencil $\mathscr{L}$ yields a conic bundle $\Psi: Y \rightarrow \mathbb{P}^{1}$ with a section, which is a component of $D$, say $\Delta_{0}$. In particular, $d \leqslant 8$. For a general fiber $L$ of $\Psi$ we have

$$
L^{2}=0, \quad-K_{Y} \cdot L=2=D \cdot L=\delta_{0} .
$$

Note that $\Psi$ has exactly $8-d$ degenerate fibers $L_{1}, \ldots, L_{8-d}$. Each of these fibers is reduced and consists of two $(-1)$-curves meeting transversally at a point. Let $C_{i}$ be the component of $L_{i}$ that meets $\Delta_{0}$. We claim that each $C_{i}$ is a component of $D$. Indeed, otherwise

$$
1=-K_{Y} \cdot C_{i}=D \cdot C_{i} \geqslant \delta_{0} \Delta_{0} \cdot C_{i}=\delta_{0}=2,
$$

which is a contradiction. Therefore we may assume that $C_{i}=\Delta_{i}$ and so

$$
1=D \cdot C_{i} \geqslant \delta_{0} \Delta_{0} \cdot C_{i}+\delta_{i} C_{i}^{2}=2-\delta_{i} .
$$

Hence $\delta_{i} \geqslant 1$ for $i=1, \ldots, 8-d$. We obtain

$$
d=-K_{Y} \cdot D \geqslant \sum \delta_{i} \geqslant \delta_{0}+\sum_{i=1}^{8-d} \delta_{i} \geqslant 2+8-d=10-d .
$$

Thus $d \geqslant 5$ as stated.

Remark 4.3. If $\operatorname{Bs} \mathscr{L}=\{P\}$ (Bs $\mathscr{L}=\emptyset$, respectively), then all the components $\Delta_{i}$ of $D$ (all the components $\Delta_{i}$ of $D$ except for $\Delta_{0}$, respectively) are contained in the fibers of $\Psi$. Indeed, otherwise not all the fibers of $\Psi \mid U$ were $\mathbb{A}^{1}$-curves, contrary to the definition of a cylinder.

Lemma 4.4. For the number $n$ of irreducible components of the curve $\operatorname{supp}(D)$ we have $n \geqslant 10-d$.

Proof. Consider the exact sequence

$$
\bigoplus_{i=1}^{n} \mathbb{Z}\left[\Delta_{i}\right] \longrightarrow \operatorname{Pic}(Y) \longrightarrow \operatorname{Pic}(U) \longrightarrow 0
$$

Since $\operatorname{Pic}(Z)=0$ and $U \cong Z \times \mathbb{A}^{1}$, we have $\operatorname{Pic}(U)=0$. Hence $n \geqslant \rho(Y)=10-d$, as stated.

Lemma 4.5. Assume that $\mathrm{Bs} \mathscr{L}=\{P\}$. Let $L$ be a member of $\mathscr{L}$ and let $C$ be an irreducible component of $L$. Then the following hold:

(i) $\operatorname{supp}(L)$ is simply connected and $\operatorname{supp}(L) \backslash\{P\}$ is an $S N C$ divisor;

(ii) $C$ is rational and smooth outside $P$;

(iii) if $P \in C$, then $C \backslash\{P\} \simeq \mathbb{A}^{1}$.

Proof. All the assertions follow from the fact that $q$ in (4.2) is a rational curve fibration and the fact that the exceptional locus of $p$ coincides with $p^{-1}(P)$.

In the next lemma we study the singularities of the pair $(Y, D)$. We refer to [Kol97] or to [KM98, Chapter 2] for the standard terminology on singularities of pairs.

Lemma 4.6 (Key Lemma). Assume that Bs $\mathscr{L}=\{P\}$. Then the pair $(Y, D)$ is not log canonical at $P$. More precisely, using the notation introduced in 4.1, the discrepancy $a(S ; D)$ of $S$ with respect to $K_{Y}+D$ is equal to -2 .

Proof. We write

$$
K_{W}+D_{W} \sim_{\mathbb{Q}} p^{*}\left(K_{Y}+D\right)+a(S ; D) S+\sum a(E ; D) E,
$$




\section{Unipotent group aCtions on Del Pezzo CONES}

where the summation on the right-hand side ranges over the components of the exceptional divisor of $p$ except for $S$, and $D_{W}$ is the proper transform of $D$ on $W$. Letting $l$ be a general fiber of $q$, by (4.3) we obtain

$$
-2=\left(K_{W}+D_{W}\right) \cdot l=a(S ; D) .
$$

Indeed, $K_{Y}+D \sim_{\mathbb{Q}} 0$ and $l$ does not meet the curve $\operatorname{supp}\left(D_{W}+p^{*}(P)-S\right)$. This proves the assertion.

Corollary 4.7. If $\operatorname{Bs} \mathscr{L}=\{P\}$, then $\operatorname{mult}_{P}(D)>1$.

Proof. Indeed, otherwise the pair $(Y, D)$ would be canonical by [Kol97, Ex. 3.14.1], and in particular, $\log$ canonical at $P$, which contradicts Lemma 4.6 .

Corollary 4.8. If Bs $\mathscr{L}=\{P\}$, then every (-1)-curve $C$ on $Y$ passing through $P$ is contained in $\operatorname{supp}(D)$.

Proof. Assume to the contrary that $C$ is not a component of $D$. Then

$$
\operatorname{mult}_{P} D \leqslant C \cdot D=-K_{Y} \cdot C=1,
$$

which contradicts Corollary 4.7.

Convention 4.9. From now on we assume that $d \leqslant 3$. By Lemma 4.2 we have Bs $\mathscr{L}=\{P\}$.

Lemma 4.10. We have $\lfloor D\rfloor=0$, that is, $\delta_{i}<1$ for all $i=1, \ldots, n$.

Proof. For the case $d=3$, see [KPZ11b, Lemma 4.1.5]. Consider the case $d=1$. By Lemma 4.4, $n \geqslant 9$. For any $i=1, \ldots, n$, we have

$$
1=-K_{Y} \cdot D=\sum_{j=1}^{n} \delta_{j}\left(-K_{Y}\right) \cdot \Delta_{j}>\delta_{i}\left(-K_{Y}\right) \cdot \Delta_{i} .
$$

Since the anticanonical divisor $-K_{Y}$ is ample, it follows that $\delta_{i}<1$, as required.

Now let $d=2$. Assuming that $\delta_{1} \geqslant 1$, we obtain

$$
2=-K_{Y} \cdot D=\sum_{i=1}^{n} \delta_{i}\left(-K_{Y}\right) \cdot \Delta_{i}>\delta_{1}\left(-K_{Y}\right) \cdot \Delta_{1} \geqslant-K_{Y} \cdot \Delta_{1},
$$

where $n \geqslant 8$ by Lemma 4.4. It follows that $-K_{Y} \cdot \Delta_{1}=1$, that is, $\Delta_{1}$ is a $(-1)$-curve. Then $C:=\tau\left(\Delta_{1}\right)$ is also a $(-1)$-curve, where $\tau$ is the Geiser involution, and $\Delta_{1}+C \sim-K_{Y}$. If $C \subset \operatorname{supp}(D)$, for example, $C=\Delta_{2}$, then by (4.4) we obtain that $\delta_{2}<1$. Now $\Delta_{1}+\Delta_{2} \sim_{\mathbb{Q}} D$ yields a relation with positive coefficients

$$
\left(1-\delta_{2}\right) \Delta_{2} \sim_{\mathbb{Q}}\left(\delta_{1}-1\right) \Delta_{1}+\sum_{i=3}^{n} \delta_{i} \Delta_{i} .
$$

This implies that $C^{2}=\Delta_{2}^{2} \geqslant 0$, which is a contradiction.

Hence $C \not \subset \operatorname{supp}(D)$. Thus $C \sim_{\mathbb{Q}} D-\Delta_{1}$, where the right-hand side is effective. This leads to a contradiction as before.

LEMMA 4.11 (cf. [KPZ11b, Lemma 4.1.6]). For a member $L$ of $\mathscr{L}$, any irreducible component of $L$ passes through the base point $P$ of $\mathscr{L}$. 


\section{Kishimoto, Prokhorov and Zaidenberg}

Proof. Assume to the contrary that there exists a component $C$ of $L$ such that $P \notin C$. Then $C^{2}<0$ (see the proof of Lemma 4.2). Since we also have $-K_{Y} \cdot C>0, C$ is a (-1)-curve. Let $C^{\prime}$ be a component of $L$ meeting $C$. If $P \notin C^{\prime}$, then $C$ and $C^{\prime}$ are both $(-1)$-curves and so $L=C+C^{\prime}$. Thus $\mathscr{L}=\left|C+C^{\prime}\right|$ is base point free, which contradicts Lemma 4.2. Hence $C^{\prime}$ passes through $P$. Since $P$ is a unique base point of $\mathscr{L}, C$ does not meet any member $L^{\prime} \in \mathscr{L}$ different from $L$. By Lemma 4.5, $L$ is simply connected, so $C^{\prime}$ is the only component of $L$ meeting $C$. Note that $\operatorname{supp}(D)$ is connected because $D$ is ample. Hence $C^{\prime}$ must be contained in $\operatorname{supp}(D)$. In fact, supposing to the contrary that $C^{\prime}$ is not contained in $\operatorname{supp}(D)$, the curve $C$ must be contained in $\operatorname{supp}(D)$. Indeed, the affine surface $U=Y \backslash \operatorname{supp}(D)$ does not contain any complete curve. Since $\operatorname{supp}(D)$ is connected, there is an irreducible component of $\operatorname{supp}(D)$ intersecting $C$ and passing through $P$. This contradicts Lemma 4.5. Thus we may suppose that $C^{\prime}=\Delta_{1}$.

If $C \subset \operatorname{supp}(D)$, say, $C=\Delta_{2}$, then

$$
1=-K_{Y} \cdot C=\left(\sum_{i=1}^{n} \delta_{i} \Delta_{i}\right) \cdot \Delta_{2}=\delta_{1}-\delta_{2} .
$$

Hence $\delta_{1}=\delta_{2}+1>1$, which contradicts Lemma 4.10.

Therefore $C \not \subset \operatorname{supp}(D)$ and so

$$
1=-K_{Y} \cdot C=\left(\sum_{i=1}^{n} \delta_{i} \Delta_{i}\right) \cdot C=\delta_{1},
$$

which again gives a contradiction by Lemma 4.10 .

\section{Proof of Theorem 1.1}

Below, we freely use the notation of the previous section. According to our geometric criterion (see Theorem 2.1), Theorem 1.1 is a consequence of the following proposition.

Proposition 5.1. Let $Y$ be a del Pezzo surface of degree $d \leqslant 2$. Then $Y$ does not admit any $\left(-K_{Y}\right)$-polar cylinder.

Convention 5.2. We let $Y$ be a del Pezzo surface of degree $d \leqslant 2$. We assume to the contrary that $Y$ possesses a $\left(-K_{Y}\right)$-polar cylinder $U$ as in (4.1). By Lemma 4.2, we have Bs $\mathscr{L}=\{P\}$.

Lemma 5.3. For any $R \in\left|-K_{Y}\right|$, we have $\operatorname{supp}(R) \not \subset \operatorname{supp}(D)$.

Proof. Suppose to the contrary that $\operatorname{supp}(R) \subset \operatorname{supp}(D)$. Let $\lambda \in \mathbb{Q}_{>0}$ be maximal such that $D-\lambda R$ is effective. We can write

$$
D=\lambda R+D_{\text {res }},
$$

where $D_{\text {res }}$ is an effective $\mathbb{Q}$-divisor such that $\operatorname{supp}(R) \not \subset \operatorname{supp}\left(D_{\text {res }}\right)$. For $t \in \mathbb{Q} \geqslant 0$, we consider the following linear combination:

$$
D_{t}:=D-t R+\frac{t}{1-\lambda} D_{\mathrm{res}} \sim_{\mathbb{Q}}-K_{Y}
$$

We have $D_{0}=D$ and $D_{\lambda}=\frac{1}{1-\lambda} D_{\text {res. }}$. For $t<\lambda$, the $\mathbb{Q}$-divisor $D_{t}$ is effective with $\operatorname{supp}\left(D_{t}\right)=$ $\operatorname{supp}(D)$. By Lemma 4.6 applied to $D_{t}$ instead of $D$, for any $t<\lambda$, the pair $\left(Y, D_{t}\right)$ is not $\log$ canonical at $P$, with discrepancy $a\left(S ; D_{t}\right)=-2$. Since the function $t \mapsto a\left(S ; D_{t}\right)$ is continuous, passing to the limit, we obtain $a\left(S ; D_{\lambda}\right)=-2$. Hence the pair $\left(Y, D_{\lambda}\right)$ is not log canonical at $P$ either and so $\operatorname{mult}_{P}\left(D_{\lambda}\right)>1$. 


\section{Unipotent Group aCtions on Del Pezzo cones}

Assume that $R$ is irreducible. Since $R \subset \operatorname{supp}(D), R$ is a component of a member of $\mathscr{L}$. Hence the curve $R$ is smooth outside $P$ and rational (see Lemma 4.5(ii)). Since $p_{a}(R)=1, R$ is singular at $P$ and $\operatorname{mult}_{P}(R)=2$. Since $R$ is different from the components of $D_{\lambda}$ and $\operatorname{mult}_{P}\left(D_{\lambda}\right)>1$, we obtain

$$
2 \geqslant K_{Y}^{2}=D_{\lambda} \cdot R \geqslant \operatorname{mult}_{P}\left(D_{\lambda}\right) \operatorname{mult}_{P}(R)>2,
$$

which is a contradiction.

Now let $R$ be reducible. By Lemma 3.4, we have $d=2$ and $R=R_{1}+R_{2}$, where, say, $R_{i}=\Delta_{i}$ for $i=1,2$ are (-1)-curves passing through $P$ (see Lemma 4.11). We may assume that $\delta_{1} \leqslant \delta_{2}$ and so $\lambda=\delta_{1}$. Since $\Delta_{1}$ is not a component of $D_{\lambda}$, we obtain

$$
1=-K_{Y} \cdot R_{1}=D_{\lambda} \cdot \Delta_{1} \geqslant \operatorname{mult}_{P}\left(D_{\lambda}\right)>1,
$$

which is a contradiction. This finishes the proof.

Proof of Proposition 5.1 in the case $d=1$. Since $\operatorname{dim}\left|-K_{Y}\right|=1$, there is a $C \in\left|-K_{Y}\right|$ passing through $P$. Furthermore, by Lemma 3.4, $C$ is irreducible. By Lemma 5.3, $C$ is not contained in $\operatorname{supp}(D)$. As in (5.1), we get a contradiction. Indeed, by Corollary 4.7, we have

$$
1=C^{2}=D \cdot C \geqslant \operatorname{mult}_{P} D \cdot \operatorname{mult}_{P} C>1 .
$$

Convention 5.4. From now on, we assume that $d=2$.

Lemma 5.5. A member $R \in\left|-K_{Y}\right|$ cannot be singular at $P$.

Proof. Assume that $P \in \operatorname{Sing}(R)$. By Lemma 3.4, we have two possibilities for $R$. Suppose first that $R$ is irreducible. By Lemma 5.3, $R \not \subset \operatorname{supp}(D)$, and we get a contradiction as in (5.1). In the second case, $R=R_{1}+R_{2}$, where $R_{1}$ and $R_{2}$ are (-1)-curves passing through $P$. Hence $R_{1}, R_{2} \subset \operatorname{supp}(D)$ by Corollary 4.8. The latter contradicts Lemma 5.3.

Notation 5.6. We let $f: Y^{\prime} \rightarrow Y$ be the blowup of $P$ and let $E^{\prime} \subset Y^{\prime}$ be the exceptional divisor. By Lemma 3.1, $Y^{\prime}$ is a weak del Pezzo surface of degree 1.

5.7. Applying Proposition 5.1 with $d=1$, we can conclude that $Y^{\prime}$ is not del Pezzo because it contains a $\left(-K_{Y}\right)$-polar cylinder. Indeed, let $D^{\prime}$ be the crepant pull-back of $D$ on $Y^{\prime}$, that is,

$$
K_{Y^{\prime}}+D^{\prime}=f^{*}\left(K_{Y}+D\right) \quad \text { and } \quad f_{*} D^{\prime}=D .
$$

Then we have

$$
D^{\prime}=\sum_{i=1}^{6} \delta_{i} \Delta_{i}^{\prime}+\delta_{0} E^{\prime}, \quad \text { where } \quad \delta_{0}=\operatorname{mult}_{P}(D)-1>0
$$

(see Lemma 4.7) and $\Delta_{i}^{\prime}$ is the proper transform of $\Delta_{i}$ on $Y^{\prime}$. Thus $D^{\prime}$ is an effective $\mathbb{Q}$-divisor on $Y^{\prime}$ such that $D^{\prime} \sim_{\mathbb{Q}}-K_{Y^{\prime}}$ and $Y^{\prime} \backslash \operatorname{supp} D^{\prime} \simeq U \simeq Z \times \mathbb{A}^{1}$ is a $\left(-K_{Y}\right)$-polar cylinder.

LEMma 5.8. We have $\operatorname{mult}_{P}(D)<2$ and $\left\lfloor D^{\prime}\right\rfloor=0$.

Proof. Suppose first that all components of $D$ are $(-1)$-curves. Then $\Delta_{i} \cdot \Delta_{j}=1$ for $i \neq j$ by Remark 3.5 and Lemma 5.3. Hence $f$ is a $\log$ resolution of the pair $(Y, D)$. Therefore $1-\sum \delta_{i}=$ $a\left(Y, E^{\prime}\right)<-1$ by Lemma 4.6, so $\sum \delta_{i}>2$. On the other hand, $2=-K_{Y} \cdot D=\sum \delta_{i}$, which is a contradiction. This shows that there exists a component $\Delta_{i}$ of $D$ which is not a (-1)-curve. By the dimension count there exists an effective divisor $R \in\left|-K_{Y}\right|$ passing through $P$ and a 


\section{Kishimoto, Prokhorov and ZaidenberG}

general point $Q \in \Delta_{i}$. On the other hand, there is no (-1)-curve in $Y$ passing through $Q$. So by Lemma 3.4, we may assume that $R$ is reduced and irreducible. By Lemma 5.3, $R$ is different from the components of $D$. Assuming that $\operatorname{mult}_{P}(D) \geqslant 2$, we obtain

$$
2=R \cdot D \geqslant \operatorname{mult}_{P}(D)+\delta_{i}>2,
$$

which is a contradiction. This proves the first assertion. The second assertion follows because $\delta_{0}>0$ in (5.2).

Corollary 5.9. The pair $\left(Y^{\prime}, D^{\prime}\right)$ is Kawamata log terminal in codimension one and is not $\log$ canonical at some point $P^{\prime} \in E^{\prime}$.

Proof. This follows from Lemma 5.8 taking into account that $D^{\prime}$ is the crepant pull-back of $D$, see [Kol97, L. 3.10].

Since $\operatorname{dim}\left|-K_{Y^{\prime}}\right|=1$, there exists an element $C^{\prime} \in\left|-K_{Y^{\prime}}\right|$ passing through the point $P^{\prime}$ as in Corollary 5.9.

Lemma 5.10. The point $P \in Y$ is a smooth point of the image $C=f_{*} C^{\prime}$.

Proof. This follows by Lemma 5.5 because $C \in\left|-K_{Y}\right|$ passes through $P$.

Corollary 5.11. $E^{\prime}$ is not a component of $C^{\prime}$.

Proof. We can write $f^{*} C=C^{\prime}+k E^{\prime}$ for some $k \in \mathbb{Z}$. Then $k=-k E^{\prime 2}=C^{\prime} \cdot E^{\prime}=1$. By Lemma 5.10, the coefficient of $E^{\prime}$ in $f^{*} C$ is equal to 1 as well. The assertion now follows.

Lemma 5.12. $C$ is reducible.

Proof. Indeed, otherwise $C^{\prime}$ is irreducible by Corollary 5.11. Since mult $P_{P^{\prime}} D^{\prime}>1$ by Corollary 5.9 and $D^{\prime} \cdot C^{\prime}=K_{Y^{\prime}}^{2}=1, C^{\prime}$ is a component of $D^{\prime}$. Hence $C$ is a component of $D$. This contradicts Lemma 5.3.

Lemma 5.13. We have $C^{\prime}=C_{1}^{\prime}+C_{2}^{\prime}$, where $C_{1}$ is a (-1)-curve, $C_{2}^{\prime}$ is a (-2)-curve, and $C_{1}^{\prime} \cdot C_{2}^{\prime}=2$. Furthermore, $P^{\prime} \in C_{2}^{\prime} \backslash C_{1}^{\prime}$ and $C_{2}=f\left(C_{2}^{\prime}\right)$ is a $(-1)$-curve.

Proof. Since $C$ is reducible and $C \in\left|-K_{Y}\right|$, by Lemma 3.4, $C=C_{1}+C_{2}$, where $C_{1}, C_{2}$ are (-1)-curves with $C_{1} \cdot C_{2}=2$. By Lemma 5.10, $P \notin C_{1} \cap C_{2}$, where $C_{2}$ is a component of $D$ by Corollary 4.8 , while by Lemma $5.3, C_{1}$ is not. So we may assume that $P \in C_{2} \backslash C_{1}$. The lemma now follows from Corollary 5.9.

5.14. Letting $C_{2}=\Delta_{1}$ from now on, we can write $D=\delta_{1} C_{2}+D_{\text {res }}$, where $\delta_{1}>0$, $D_{\text {res }}$ is an effective $\mathbb{Q}$-divisor, and $C_{2}$ is not a component of $D_{\text {res }}$. Similarly,

$$
D^{\prime}=\delta_{1} C_{2}^{\prime}+D_{\text {res }}^{\prime}+\delta_{0} E^{\prime},
$$

where $D_{\text {res }}^{\prime}$ is the proper transform of $D_{\text {res }}$ and $\delta_{0}=\operatorname{mult}_{P}(D)-1$ (cf. (5.2)).

Lemma 5.15. We have $2 \delta_{1} \leqslant 1$.

Proof. This follows from

$$
0 \leqslant D_{\text {res }} \cdot C_{1}=\left(D-\delta_{1} C_{2}\right) \cdot C_{1}=1-2 \delta_{1} .
$$

Lemma 5.16. In the same notation as before, $\delta_{0}+D_{\text {res }}^{\prime} \cdot C_{2}^{\prime}>1$. 


\section{Unipotent group aCtions on Del PeZzo CONES}

Proof. Let us show first that $\left\{P^{\prime}\right\}=C_{2}^{\prime} \cap E^{\prime}=C_{2}^{\prime} \cap \operatorname{supp}\left(D_{\text {res }}^{\prime}\right)$. Indeed, $P^{\prime} \in E^{\prime}$ by construction, $P^{\prime} \in C_{2}^{\prime}$ by Lemma 5.13, and $P^{\prime} \in \operatorname{supp}\left(D_{\text {res }}^{\prime}\right)$ because otherwise $P^{\prime}$ would be a node of $D^{\prime}$ (indeed, $E^{\prime}$ meets $C_{2}^{\prime}$ transversally at $\left.P^{\prime}\right)$ and so the pair $\left(Y^{\prime}, D^{\prime}\right)$ would be $\log$ canonical at $P^{\prime}$, contrary to Corollary 5.9. On the other hand, the curves $C_{2}^{\prime}$ and $D_{\text {res }}^{\prime}$ have only one point in common, by Lemma 4.5(i).

Since $\delta_{1}<1$, the pair $\left(Y^{\prime}, C_{2}^{\prime}+D_{\text {res }}^{\prime}+\delta_{0} E^{\prime}\right)$ is not log canonical at $P^{\prime}$. By applying [KM98, Corollary 5.57], we now obtain

$$
1<\left(D_{\mathrm{res}}^{\prime}+\delta_{0} E^{\prime}\right) \cdot C_{2}^{\prime}=\delta_{0}+D_{\mathrm{res}}^{\prime} \cdot C_{2}^{\prime},
$$

as stated.

Proof of Proposition 5.1 in the case $d=2$. We use the same notation as above. Since $C_{2}^{\prime}$ is a (-2)-curve, by virtue of Lemmas 5.15 and 5.16 , we have

$$
1-\delta_{0}<D_{\mathrm{res}}^{\prime} \cdot C_{2}^{\prime}=\left(D^{\prime}-\delta_{1} C_{2}^{\prime}-\delta_{0} E^{\prime}\right) \cdot C_{2}^{\prime}=2 \delta_{1}-\delta_{0} \leqslant 1-\delta_{0},
$$

which is a contradiction. Now the proof of Proposition 5.1 is completed.

Remark 5.17. Our proof of Proposition 5.1 goes along the lines of that of Lemmas 3.1 and 3.5 in [Chel08]. ${ }^{1}$ However, this proposition does not follow immediately from the results in [Chel08]. Indeed, in the notation of [Chel08], by Lemma 4.6, we have $\operatorname{lct}(Y, D)<1$. This is not sufficient to get a contradiction with [Chel08, Theorem 1.7]. The point is that our boundary $D$ is not arbitrary, on the contrary, it is rather special (see Lemma 4.5).

\section{Acknowledgements.}

This work was done during a stay of the second and third authors at the Max Planck Institute für Mathematik at Bonn and a stay of the first and the second authors at the Institute Fourier, Grenoble. The authors thank these institutions for their hospitality and support. They are also grateful to the referee for useful comments.

\section{REFERENCES}

Chel08 I. Cheltsov, Log canonical thresholds of del Pezzo surfaces, Geom. Funct. Anal. 18 (2008), no. 4, 1118-1144. http://dx.doi.org/10.1007/s00039-008-0687-2

CPW13 I. Cheltsov, J. Park and J. Won, Affine cones over smooth cubic surfaces, arXiv:1303.2648 (2013).

Dol12 I. Dolgachev, Classical Algebraic Geometry: A Modern View, Cambridge University Press, Cambridge, 2012.

FZ03 H. Flenner and M. Zaidenberg, Rational curves and rational singularities, Math. Z. 244 (2003), no. 3, 549-575.

KM98 J. Kollár and S. Mori, Birational geometry of algebraic varieties, With the collaboration of C. H. Clemens and A. Corti, Translated from the 1998 Japanese original, Cambridge Tracts in Mathematics 134, Cambridge University Press, Cambridge, 1998.

Kol97 J. Kollár, Singularities of pairs, In Algebraic geometry-Santa Cruz 1995, Proc. Sympos. Pure Math. 62, 221-287, Amer. Math. Soc. Providence, RI, 1997.

KPZ11a T. Kishimoto, Yu. Prokhorov and M. Zaidenberg, Affine cones over Fano threefolds and additive group actions, Osaka J. Math. (to appear), arXiv:1106.1312 (2011).

\footnotetext{
${ }^{1}$ We are grateful to Ivan Cheltsov, who attracted our attention to this fact.
} 


\section{Kishimoto, Prokhorov and Zaidenberg}

KPZ11b T. Kishimoto, Yu. Prokhorov and M. Zaidenberg, Group actions on affine cones, In Affine algebraic geometry, 123-163. Peter Russell's Festschrift, CRM Proc. Lecture Notes, 54, Amer. Math. Soc. Providence, RI, 2011.

KPZ12 T. Kishimoto, Yu. Prokhorov and M. Zaidenberg, $\mathbf{G}_{a}$-actions on affine cones, Transf. Groups (to appear), arXiv:1212.4249 (2012).

Per11 A. Perepechko, Flexibility of affine cones over del Pezzo surfaces of degree 4 and 5, Funct. Anal. Appl. (to appear), arXiv:1108.5841 (2011).

Takashi Kishimoto tkishimo@rimath.saitama-u.ac.jp

Department of Mathematics, Faculty of Science, Saitama University, Saitama 338-8570, Japan

Yuri Prokhorov prokhoro@gmail.com

Steklov Institute of Mathematics, 8 Gubkina street, Moscow 119991, Russia

and

Laboratory of Algebraic Geometry, GU-HSE, 7 Vavilova street, Moscow 117312, Russia

Mikhail Zaidenberg zaidenbe@ujf-grenoble.fr

Université Grenoble I, Institute Fourier, UMR 5582 CNRS-UJF, BP 74, 38402 Saint Martin d'Heres cedex, France 